\title{
Outbreak of novel coronavirus (COVID-19): What is the role of radiologists?
}

\author{
Hyungjin Kim ${ }^{1}$
}

Received: 12 February 2020 / Accepted: 13 February 2020 /Published online: 18 February 2020

(C) European Society of Radiology 2020

\section{Key Points}

- Novel coronavirus (COVID-19)-infected pneumonia usually manifests as bilateral ground-glass opacities in the lung periphery on chest CT scans.

- Role of radiologists includes not only early detection of lung abnormality, but also suggestion of disease severity, potential progression to acute respiratory distress syndrome, and possible bacterial co-infection in hospitalized patients.

Novel coronavirus (COVID-19) was first reported from Wuhan, China in December 2019. It was rapidly spread to other regions of China, to the neighboring countries including Singapore, Thailand, South Korea, Japan, and Malaysia, and even to a few Western countries. As of February 11, the total number of the reported cases is 43,112 [1], which far exceeded the number of patients with severe acute respiratory syndrome (SARS). According to Wu et al. [2], basic reproduction number of COVID-19 was estimated to be 2.68 (95\% confidence interval, 2.47-2.86), which indicates that more than two new cases are generated by a single infected patient. The estimated reproduction numbers of SARS and middle east respiratory syndrome (MERS) ranged from 2 to 5 and from 2.7 to 3.9, respectively [3]. For the patient outcome, Huang et al. [4] initially reported that mortality rate of COVID-19 was $15 \%$ (6/41). However, later studies reported lower mortality rates from $4.3 \%$ to $11 \%[5,6]$. According to an online dashboard developed by the Johns Hopkins Center for Systems Science and Engineering, the mortality rate is 2.4\% (1018/43112) as of February 11 and the mortality rate outside China is much lower $(0.4 \% ; 2 / 464)[1]$. The mortality rates of SARS and MERS were $9.5 \%$ and $34.4 \%$, respectively [7].

For the radiological findings, Song et al. [8] summarized $\mathrm{CT}$ findings of 51 patients and reported that the most frequent CT feature was ground-glass opacity (GGO; $77 \%$ ) followed by GGO with reticular and/or interlobular septal thickening

Hyungjin Kim

khj.snuh@gmail.com

1 Department of Radiology, Seoul National University Hospital, 101, Daehak-ro, Jongno-gu, Seoul 03080, South Korea
(75\%). Most of the patients (86\%) had bilateral lung involvement. Younger patients ( $\leq 50$ years) tended to have more GGOs, while older patients ( $>50$ years) had more consolidations with organizing pneumonia pattern. Michael et al. [9] published a case series of 21 patients. In that study, GGO was also the most frequent manifestation (57\%) and most of the patients (76\%) had a bilateral disease. Interestingly, 33\% had a round GGO and/or consolidation for their initial manifestation. General radiological features of COVID-19 are quite similar to those of SARS. It is not surprising given the fact that SARS is also caused by a strain of coronavirus, which has genetic similarity with COVID-19.

To date, little is known for the temporal radiological change of COVID-19 pneumonia, but it can be inferred from the past experience. In patients with SARS, lung lesions detected in the first week from the symptom onset aggravated in the second week [10]. Then, the lesions changed into irregular reticular opacities mixed with GGOs at the fourth week. Similarly, Pan et al. [11] described that $85.7 \%$ (54/63) of their cohort with COVID-19 pneumonia showed disease progression (i.e., increased extent of GGO and consolidation) at early follow-up CT scans (interval, 3-14 days). Lung fiber cord was found in a patient with improvement as the inflammation was absorbed [12]. The long-term sequelae in patients with severe pneumonia, although not available for the present, could be the fibrotic changes such as reticulation, interlobular septal thickening, and traction bronchiectasis, which are usually seen in the fibrotic phase of lung injury.

To be noted, there is a report of asymptomatic pneumonia among patients with COVID-19 infection [13]. That is, a subset of patients can be asymptomatic even with pneumonic changes in lungs. Thus, care should be taken for identification of such atypical patients as these may be sources of community 
transmission. Understandably, patients with COVID-19 infection can also have negative chest radiographs or CT scans.

There are several roles of radiologists in this outbreak. First, early detection of the radiological abnormality can provide a suspicion of pneumonia in patients at risk. Although, the definitive diagnosis of COVID-19 infection is based on reverse transcription polymerase chain reaction, radiological findings are important for the diagnosis of pneumonia. Timely diagnosis of COVID-19 pneumonia would enable rapid management planning including further imaging follow-ups and conservative care. Given the fact that the most frequent imaging finding is GGO, careful interpretation of the chest radiographs is needed as GGOs may look very faint. CT scans are recommended in patients with suspicious lung abnormality. Second, disease severity can be suggested and notified to the clinicians. Michael et al. [9] reported that one COVID-19-infected patient with the highest severity score (lung involvement score) on the CT scan was admitted to the intensive care unit. In addition, a high rate (19.6\%) of acute respiratory distress syndrome (ARDS) was observed from the patients with COVID-19 pneumonia [5]. Imaging plays an important role in the diagnosis and follow-up of ARDS. Third, radiologists can give a suspicion of bacterial secondary infection. Notably, bacterial co-infection was common (12\%-19\%) among hospitalized patients with pandemic H1N1 influenza and confirmed pneumonia [14]. Bacterial co-infection was associated with serious outcomes [14]. Therefore, it is our role to provide an impression of secondary infection, which would necessitate antibiotic treatment. Fourth, radiologists should make an effort to educate trainees and clinicians of the imaging findings of emerging infectious diseases including COVID-19. This is the third outbreak caused by the coronavirus in the recent decades. Radiologists and clinicians should be prepared for unexpected infectious diseases to fight epidemic and potential pandemic.

Lastly, new weapons we have in this decade are the artificial intelligence (AI) models for imaging studies. Diagnostic AI models would enable prompt risk prioritization and help reduce turnaround time. Such models would be extremely useful in a mass screening setting such as the outbreak in China. Given the shortage of manpower and hospital beds in China, AI models for the chest radiographs and CT scans may help alleviate the burden of radiologists and clinicians and enhance rapid triaging.

Funding information The author states that this work has not received any funding.

\section{Compliance with ethical standards}

Guarantor The scientific guarantor of this publication is Hyungjin Kim.

Conflict of interest Activities related to the present article: none.

Activities not related to the present article: H.K. received a research grant from Lunit Inc. (Seoul, South Korea).
Statistics and biometry Not applicable

Informed consent Not applicable

Ethical approval Not applicable

Study subjects or cohorts overlap Not applicable

Methodology Not applicable

\section{References}

1. Johns Hopkins Center for Systems Science and Engineering (2020) Coronavirus COVID-19 global cases. Available via https:// gisanddata.maps.arcgis.com/apps/opsdashboard/index.html\#/ bda7594740fd40299423467b48e9ecf6. Accessed 11 Feb 2020

2. Wu JT, Leung K, Leung GM (2020) Nowcasting and forecasting the potential domestic and international spread of the 2019-nCoV outbreak originating in Wuhan, China: a modelling study. Lancet. https://doi.org/10.1016/S0140-6736(20)30260-9

3. Zhao S, Lin Q, Ran J et al (2020) Preliminary estimation of the basic reproduction number of novel coronavirus (2019-nCoV) in China, from 2019 to 2020: a data-driven analysis in the early phase of the outbreak. Int J Infect Dis. https://doi.org/10.1016/j.ijid.2020.01.050

4. Huang C, Wang Y, Li X et al (2020) Clinical features of patients infected with 2019 novel coronavirus in Wuhan, China. Lancet. https://doi.org/10.1016/S0140-6736(20)30183-5

5. Wang D, Hu B, Hu C et al (2020) Clinical characteristics of 138 hospitalized patients with 2019 novel coronavirus-infected pneumonia in Wuhan, China. JAMA. https://doi.org/10.1001/jama.2020.1585

6. Chen N, Zhou M, Dong X et al (2020) Epidemiological and clinical characteristics of 99 cases of 2019 novel coronavirus pneumonia in Wuhan, China: a descriptive study. Lancet. https://doi.org/10.1016/ S0140-6736(20)30211-7

7. Munster VJ, Koopmans M, van Doremalen N, van Riel D, de Wit E (2020) A novel coronavirus emerging in China - key questions for impact assessment. N Engl J Med. https://doi.org/10.1056/ NEJMp2000929

8. Song F, Shi N, Shan F et al (2020) Emerging coronavirus 2019nCoV pneumonia. Radiology. https://doi.org/10.1148/radiol. 2020200274:200274

9. Chung M, Bernheim A, Mei X et al (2020) CT imaging features of 2019 novel coronavirus (2019-nCoV). Radiology. https://doi.org/ 10.1148/radiol.2020200230:200230

10. Ooi GC, Khong PL, Muller NL et al (2004) Severe acute respiratory syndrome: temporal lung changes at thin-section CT in 30 patients. Radiology 230:836-844

11. Pan Y, Guan H, Zhou S et al (2020) Initial CT findings and temporal changes in patients with the novel coronavirus pneumonia (2019nCoV): a study of 63 patients in Wuhan, China. Eur Radiol. https:// doi.org/10.1007/s00330-020-06731-X

12. Pan Y, Guan H (2020) Imaging changes in patients with 2019nCov. Eur Radiol. https://doi.org/10.1007/s00330-020-06713-Z

13. Chan JF, Yuan S, Kok KH et al (2020) A familial cluster of pneumonia associated with the 2019 novel coronavirus indicating person-to-person transmission: a study of a family cluster. Lancet. https://doi.org/10.1016/S0140-6736(20)30154-9

14. MacIntyre CR, Chughtai AA, Barnes M et al (2018) The role of pneumonia and secondary bacterial infection in fatal and serious outcomes of pandemic influenza a(H1N1)pdm09. BMC Infect Dis 18:637 